\title{
Common sense gun policy reforms for the United States
}

The authors of this Editorial have informed us of an error in the eighth paragraph (BMJ 2012;345:e8672, doi:10.1136/bmj. e8672). The third sentence reads: "Only five US states set minimum age requirements for firearm possession above that of federal law," when, in fact, this is not accurate, nor was it true at the time of the article's publication. Presently, twelve
US states set minimum age requirements for firearm possession above that of federal law.

Cite this as: BMJ 2014;348:g1459

๑ BMJ Publishing Group Ltd 2014 